\title{
GIANT CELL ARTERITIS PRESENTING AS PYREXIA OF UNKNOWN ORIGIN IN
} ASIANS: 2 CASE REPORTS.

Dr Charlotte Tan SL, Dr Lee Chun-Tsu, Dr Matthew Chen ZX, Dr Nga Min En, Dr Teng Gim Gee

\begin{abstract}
Giant cell arteritis (GCA) has a Caucasian preponderance and mainly affects persons older than 50 years old. We report on 2 elderly patients of Asian origin who presented with pyrexia of unknown origin to highlight the rare occurrence of GCA in Asians without classical symptoms. Due to its wide spectrum of manifestations and ethnic differences in epidemiology, the disease can pose a diagnostic challenge to unwary clinicians, resulting in serious sequelae; thus a high index of suspicion is needed for prompt diagnosis and treatment. Temporal artery biopsy should be performed when GCA is suspected.
\end{abstract}

Keywords: Giant Cell Arteritis; Elderly; Asians; Pyrexia of Unknown Origin; Temporal Artery Biopsy;

SFP20I7; 43(4): 37-39

\section{CASE I}

A 69-year-old Indian lady presented with 3 weeks of fever, lethargy, and "clogged" sensation in her right ear associated with pain in the molar region of the oral cavity. There was no jaw claudication, headache, ear discharge, visual or hearing disturbance, proximal weakness, joint pain, or rash. She was febrile at $38^{\circ} \mathrm{C}$ with right tragus tenderness. Otoscopy and the rest of the examination were unremarkable, with no temporal region tenderness or palpable temporal arteries. Investigations showed normal leukocyte count, normochromic normocytic anaemia with haemoglobin $11.2 \mathrm{~g} / \mathrm{dL}(12.0-15.1 \mathrm{~g} / \mathrm{dL})$ and mild thrombocytosis $421 \times 109 / \mathrm{L}\left(150-300 \times 10^{9} / \mathrm{L}\right)$. Liver and renal function tests were normal. She was treated with oral co-amoxiclav for suspected otitis media, given the ear discomfort and fever. A week later, further investigations done

\section{CHARLOTTE TAN SL}

University Medicine Cluster, Division of Rheumatology,

National University Health System, Singapore

\section{LEE CHUN-TSU}

Department of Haematology-Oncology,

National University Cancer Institute,

National University Hospital, Singapore

\section{MATTHEW CHEN ZX}

University Medicine Cluster, Division of Geriatric Medicine,

National University Health System, Singapore.

\section{LEE CHUN-TSU}

Department of Haematology-Oncology,

National University Cancer Institute,

National University Hospital, Singapore

\section{TENG GIM GEE}

University Medicine Cluster, Division of Rheumatology,

National University Health System, Singapore,

Department of Medicine, Yong Loo Lin School of Medicine,

National University of Singapore, Singapore for persistent pyrexia showed an elevated erythrocyte sedimentation rate (ESR) $138 \mathrm{~mm} /$ hour $(<20 \mathrm{~mm} /$ hour$)$ and C-reactive protein (CRP) $55 \mathrm{mg} / \mathrm{L}(0-10 \mathrm{mg} / \mathrm{L})$. Given the persistence of symptoms despite antibiotics, age, and markedly elevated inflammatory markers, giant cell arteritis (GCA) was suspected. She underwent emergent temporal artery biopsy (TAB) and commenced on prednisolone $1 \mathrm{mg} / \mathrm{kg} /$ day. Her symptoms resolved and inflammatory markers normalised. The histology confirmed the diagnosis of GCA.

\section{CASE 2}

An 89-year-old Chinese lady presented with 1 day of fever, cough, and breathlessness on a background of well-controlled asthma. She was treated for infective exacerbation of asthma with oral co-amoxiclav and prednisolone $30 \mathrm{mg}$ daily for 5 days. Four days later, she was admitted for recurrence of fever with vomiting. Investigations were remarkable for leukocytosis of $18.9 \times 10^{9} / \mathrm{L}\left(3.4-9.6 \times 10^{9} / \mathrm{L}\right)$, microcytic anaemia with haemoglobin $10.4 \mathrm{~g} / \mathrm{dL}$ and thrombocytosis of $528 \times 10^{9} / \mathrm{L}$. ESR was $114 \mathrm{~mm} /$ hour and CRP was $192 \mathrm{mg} / \mathrm{L}$. Repeat chest radiograph was normal. Treatment for presumptive infective asthma exacerbation was re-prescribed. However, fevers ranging from $38.0^{\circ}$ to $39.2^{\circ} \mathrm{C}$ persisted the next 3 weeks with anorexia and weight loss of $6 \mathrm{~kg}$. There was no rash, photosensitivity, oral ulcers, proximal weakness, body aches, joint stiffness or pains, or visual disturbances. Whole-body positron-emission-tomography (PET); blood and sputum bacterial, mycobacterial, and fungal cultures; together with mycoplasma, rickettsial, retroviral serologies were negative. Her anti-nuclear antibody (ANA) titre was 1:640 and anti-extractable nuclear antigen antibodies were negative. Colonoscopy and gastroscopy were unremarkable. Bone marrow biopsy revealed reactive bone marrow. At this juncture, infections and malignancies were excluded, leaving a systemic inflammatory disorder such as polymyalgia rheumatica as the working diagnosis, thus prednisolone $20 \mathrm{mg}$ daily was commenced. The patient defervesced and her appetite returned. Due to her marked improvement, the prednisolone was tapered to $10 \mathrm{mg}$ daily after 2 weeks while she underwent rehabilitation. Six weeks later, she reported acute loss of right vision which started with intermittent temporal field loss, evolving to affect the entire visual field. She also developed severe myalgia resulting in difficulty getting out of bed. Ophthalmologic examination revealed visual acuity only to light in the right eye, with right relative afferent pupillary defect. Fundoscopy was negative for optic disc swelling or pallor and the patient was assessed to have posterior ischaemic optic neuropathy. The diagnosis of GCA was unequivocal when the patient developed sudden visual impairment and girdle muscle aches. Given her marked visual impairment, she was immediately treated with intravenous methylprednisolone $1 \mathrm{~g}$ daily for 3 days with aspirin. An emergent TAB confirmed 
the diagnosis (Figure 1). Her visual acuity improved to $6 / 120$ on the 3rd day of methylprednisolone.

\section{Figure I}

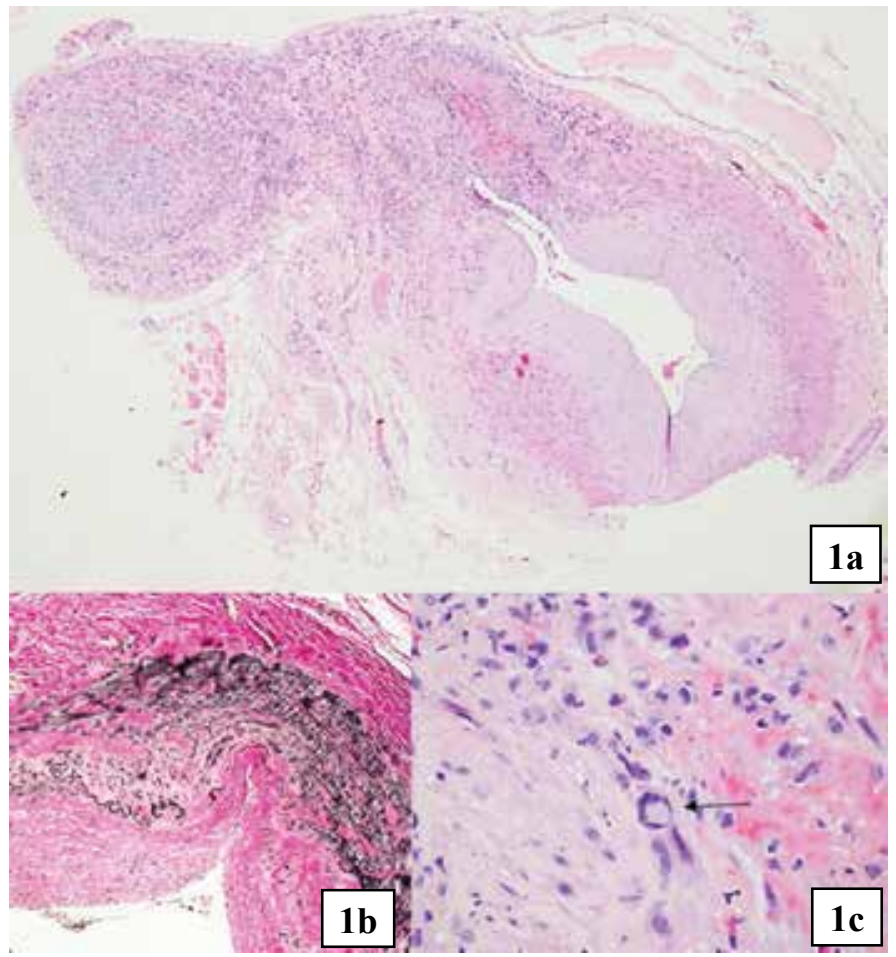

Figure 1a: Low power microscopy showing the cross section of the temporal artery with medial inflammation (Haematoxylin and Eosin, original magnification x 40) 1b: Elastic stain showing disruption of internal elastic lamina (Verhoeff-Van Gieson, original magnification x 200). 1c: High magnification view of the tunica media showing inflammatory cells including a multinucleated giant cell (arrow).

\section{DISCUSSION}

GCA does occur in Asians despite being known as a "Caucasian" disease, with an estimated incidence between 0.09-1.5 per 100000 in the USA. ${ }^{1}$ Both our patients posed diagnostic dilemmas with pyrexia of unknown origin (PUO). Common manifestations in Asians include constitutional symptoms, headaches, visual impairment, jaw claudication, scalp tenderness, and concurrent polymyalgia rheumatica — with fever and headache being the most common. ${ }^{2}$ These symptoms are similar to those for Westerners, except for a higher prevalence of constitutional symptoms described in Asians. ${ }^{2}$ Rheumatic diseases account for 25 to 30 percent of causes of PUO in the elderly and GCA is the most frequent diagnosis. ${ }^{3}$ Hence a comprehensive search for infective and neoplastic causes of PUO should not delay the pursuit of GCA diagnosis, even in regions of low disease prevalence.

Both patients had prolonged symptoms of systemic inflammation and elevated ESR/ CRP without classic symptoms like headaches, jaw claudication, scalp tenderness and visual impairment which would promptly raise suspicion of GCA. The first patient presented with tragus tenderness and tooth discomfort - such orofacial presentations, although rare, have previously been reported. ${ }^{4,5}$ The second patient had no cranial vascular features or polymyalgia until much later. Other atypical presentations include abdominal pain ${ }^{6}$ and dry cough. ${ }^{7}$ This highlights that GCA presents with protean manifestations, sometimes asynchronously. Non-specific symptoms like diffuse myalgia and anorexia may be dismissed as age-related poor health in the elderly. Retrospectively, the TAB should have been considered earlier in the second case, at the point when the PUO still posed a diagnostic dilemma in an elderly where ESR was markedly elevated. Pathognomonic histology of GCA are multinucleated giant cells and fragmented internal elastic membrane on a background of lymphocyte and macrophage infiltration most pronounced in the tunica media.

TAB has a false negative rate of up to 44 percent. ${ }^{8}$ To improve the diagnostic yield, biopsy of the contralateral temporal artery was previously practiced when the initial TAB was negative. However given that only 5 percent of the contralateral biopsy returned positive ${ }^{9}$ and with its invasive nature, imaging modalities are increasingly being used to diagnose GCA. ${ }^{10}$ Recent studies suggest the validity of color Doppler ultrasound (CDS) in diagnosing GCA. ${ }^{11}$ A systematic review by Buttgereit et al showed a modest sensitivity (55-100\%) and specificity (78-100\%) of CDS. ${ }^{12}$ The heterogeneous findings are due to different inclusion criteria, technical equipment, vessels scanned and various diagnostic standards. However, it offers the advantage of being safe, well tolerated and allows examination of the full-length superficial temporal artery and other accessible cranial or extracranial vessels. PET may also serve as an important adjunctive modality for extracranial involvement and biopsy negative GCAs. Recent meta-analysis showed that PET has high sensitivity (85\%) and specificity (91\%). ${ }^{13}$ Pitfalls include lack of standard protocols and scoring of PET, and low reliability in distinguishing GCA-mimickers like atherosclerosis and smoldering occult disease. ${ }^{13}$ In Case 2, PET may have been insensitive in picking up cranial GCA. ${ }^{13}$ Furthermore, GCA occurs without extracranial involvement in up to 85 percent of cases. ${ }^{14}$ Alternatively, PET could have been truly negative at the early stages of our patient's disease where PUO was the only manifestation. Therefore, it is suggested that CDS be considered in every patient with clinically suspected GCA, and PET for the evaluation of suspected extracranial large vessel vasculitis. If the diagnosis of GCA cannot be confirmed or ruled out by clinical, laboratory, and imaging findings, a TAB should be performed. ${ }^{12}$

\section{CONCLUSION}

A high clinical index of suspicion of GCA is necessary when elderly patients present with non-specific symptoms of systemic inflammation, even in areas of low disease prevalence such as Asia. TAB should be pursued early when GCA is suspected so that prompt treatment can be initiated to prevent serious complications developing. 


\section{REFERENCES}

I. Pereira LS, Yoon MK, Hwang TN, Hong JE, Ray K, Porco T, et al. Giant cell arteritis in Asians: a comparative study. Br J Ophthalmol. 2011;95:214-6.

2. Sun F, Ma S, Zheng W, Tian X, Zeng X. A retrospective study of Chinese patients with giant cell arteritis (GCA): clinical features and factors associated with severe ischemic manifestations. Medicine (Baltimore). 2016;95:e3213.

3. Knockaert DC, Vanneste LJ, Bobbaers HJ. Fever of unknown origin in elderly patients. J Am Geriatr Soc. 1993;41: I I87-92.

4. Vasudevan S, Palle AR, Sylvia DS, Renuka V, Challa R. A rare case of temporal arteritis with rheumatoid arthritis and interstitial lung disease mimicking pulpo-periodontal pathology. J Indian Soc Periodontol.

20|4;|8:53|-5.

5. Imran TF, Helfgott S. Respiratory and otolaryngologic manifestations of giant cell arteritis. Clin Exp Rheumatol. 20I5;33:164-70.

6. Shambhu S, Suarez L. Giant cell arteritis: an atypical presentation diagnosed with the use of MRI imaging. Case Rep Rheumatol. 2016;2016:8239549. http://dx.doi.org//0.1 I55/2016/8239549. [Accessed 08 Aug 2017].

7. Zenone T, Puget M. Dry cough is a frequent manifestation of giant cell arteritis. Rheumatol Int. 2013;33:2165-8.

8. Ashton-Key MR, Gallagher PJ. False-negative temporal artery biopsy. Am J Surg Pathol. 1992;16:634-5.

9. Pless M, Rizzo JF, 3rd, Lamkin JC, Lessell S. Concordance of bilateral temporal artery biopsy in giant cell arteritis. J neuroophthalmol. 2000;20:216-8

10. Davies CG, May DJ. The role of temporal artery biopsies in giant cell arteritis. Ann R Coll Surg Engl. 201 I;93:4-5.

II. Monti S, Floris A, Ponte C, Schmidt WA, Diamantopoulos AP, Pereira C, et al. The use of ultrasound to assess giant cell arteritis: review of the current evidence and practical guide for the rheumatologist. Rheumatology (Oxford). 2017. doi: 10.1093/rheumatology/kexI73. [Accessed 08 Aug 2017].

12. Buttgereit F, Dejaco C, Matteson EL, Dasgupta B. Polymyalgia rheumatica and giant cell arteritis: a systematic review. JAMA. 2016;3।5:2442-58.

13.Zvan der Schaft N, Compagne K, Groenendijk A, Vis M. The role of positron-emission tomography in the diagnosis of giant cell arteritis. Erasmus Journal of Medicine. 2015;5:10-6.

14.Puppo C, Massollo M, Paparo F, Camellino D, Piccardo A, Shoushtari Zadeh Naseri M, et al. Giant cell arteritis: a systematic review of the qualitative and semiquantitative methods to assess vasculitis with $18 \mathrm{~F}$-fluorodeoxyglucose positron emission tomography. BioMed Research International. 2014;2014:574248. http://dx.doi.org/I 0.I I55/20 I4/574248. [Accessed 08 Aug 20I7]. 